\title{
PHOTOLUMINESCENCE, REFLECTIVITY AND RAMAN INVESTIGATIONS OF NANOCRYSTALLITES IN LUMINESCENT POROUS SILICON*
}

\author{
W. Bala, G. Glowacki, Z. Eukasiak \\ Institute of Physics, N. Copernicus University, Grudziądzka 5, 87-100 Toruń, Poland \\ M. DrozdowsKi, M. KozielsKI \\ Institute of Physics, Poznan University of Technology \\ Piotrowo 3, 60-965 Poznań, Poland \\ E. Nossarzewska-Orlowska and A. Brzozowski
}

Institute of Electronic Materials Technology, Wólczyńska 133, 01-919 Warszawa, Poland

\begin{abstract}
Raman scattering, reflectivity and photoluminescence measurements of the porous silicon layers prepared on (001) $p / p^{+}$silicon epitaxial wafers by anodization method are presented. We have studied dependence of the frequency shift and halfwidth of LO mode in Raman spectra and shift of the luminescence peak in photoluminescence spectra vs. anodization conditions.

PACS numbers: $78.30 . \mathrm{Hv}, 78.66 .-\mathrm{w}$
\end{abstract}

There has been a considerable interest in the physical properties of porous silicon prepared by anodization method [1-6]. In particular, porous silicon layers are attractive for the possible efficient light-emitting applications in the visible region. It opens the door for silicon as a possible material for optoelectronic applications. In the present time the basic requirement for the preparation of porous silicon layers is to obtain a good quality, stable layer on Si substrate emitting efficient luminescence.

In this paper the Raman scattering (RS), reflectivity (R), photoluminescence (PL) and photoluminescence excitation (PLE) measurements of the porous silicon layers prepared on (001) $p / p^{+}$silicon epitaxial wafers by anodization method are presented.

*This work is supported in part by the State Committee for Scientific Research (Republic of Poland) under grant No. 8S501 00605 (Warszawa), grant UMK 378F (Torun), and Research Programme of Poznań University of Technology No. 62100 (Poznani). 
The porous silicon samples were prepared by the electrochemical anodization of $p$-type epitaxial layer deposited on low resistivity $p^{+}$substrate $(0.01 \Omega \mathrm{cm})$ under a current density of $10 \mathrm{~mA} / \mathrm{cm}^{2}$. As an electrolyte the $\mathrm{HF}$ acid $(40 \%$ in $\mathrm{H}_{2} \mathrm{O}$ ) diluted in isopropyl alcohol was used. The $\mathrm{HF}$ concentration was changed in the range of $45 \mathrm{wt} . \%$ to $10 \mathrm{wt} . \%$ [7]. All measurements (RS, R and PL) were carried out at room temperature. Near backscattering Raman measurements were performed using $457.9 \mathrm{~nm}$ line of an $\mathrm{Ar}^{+}$laser as an excitation source. The laser power was $10 \mathrm{~mW}$ and spot size on the samples was about $200 \mu \mathrm{m}$. The thermal effect due to laser heating of the sample was negligible. Reflectivity measurements were performed in backscattering geometry by using a $100 \mathrm{~W}$ tungsten-halogen lamp as a light source and SPM-2 monochromator equipped with R-1333 photomultiplier and photon counting system. Photoluminescence was excited with $325 \mathrm{~nm}$ radiation from $\mathrm{He}-\mathrm{Cd}$ laser or a high pressure mercury lamp. Corrected PLE spectra were recorded on a single-photon spectrofluorometer working in the ratio mode and consisting of two monochromators (SPM-2), xenon arc and a photomultiplier (R-928). Time-resolved PL experiments were carried out by excitation of the samples with a pulse nitrogen laser. The proper band of the emission was selected by a double grating monochromator and registered by a combination of photomultiplier (EMI 9558) and boxcar averager (162/164 PAR).
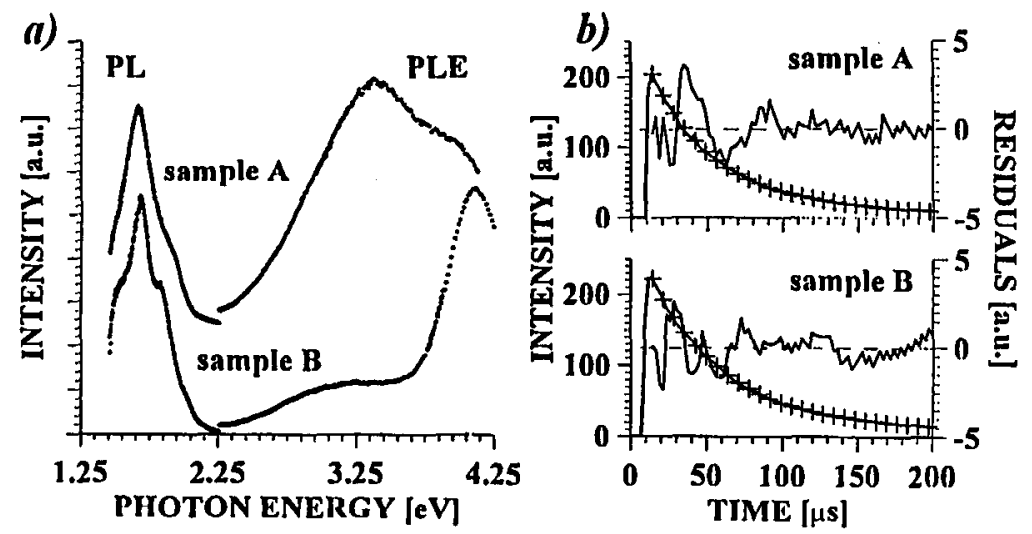

Fig. 1. PL and PLE spectra (a) and PL decay (b) for samples preparing at different anodization conditions. Sample A: HF concentration - $22 \%$, porosity $-68 \%$; sample B: HF concentration $-18 \%$, porosity $-75 \%$. Result of the fit of a multiexponential model (points) to measured decay (solid line) (b). Residuals are plotted across the picture. Decay times are in $\mu$ s.

Figure la shows representative PL and PLE spectra of the samples A and $B$ preparing from the same wafer under different anodization conditions (with porosity $68 \%$ (sample A) and $75 \%$ (sample B)). The PL spectra under mercury (or xenon) arc excitation reveal the structure which is not seen under laser illumination (low and high excitation, respectively). For the same samples at low level excitation the PL spectra exhibit multipeak luminescence. For all our samples the 
spectrum can be fitted as a sum of few gaussians. The width and the position of the luminescence peaks are determined by the relative intensity of the individual components which depends on anodization conditions [7]. The relative intensity of the luminescence peaks strongly depends on the temperature, while their position remains almost constant. As can be seen in Fig. 1a, the PL spectra are dominated by three peaks $(2.06,1.86$ and $1.72 \mathrm{eV})$. Energetical positions of these peaks do not exhibit a blue-shift with decreasing temperature. Figure 1a shows the PLE spectra from the samples A and B measured at $2.06 \mathrm{eV}$. A significant increase in the PLE signal in these samples occurs at $2.95 \mathrm{eV}$ and $3.4 \mathrm{eV}$ (sample A) and at $4.1 \mathrm{eV}$ (sample B).

Figure $1 \mathrm{~b}$ shows the example of the measured PL decay curves $D(i)$ fitted by biexponential model $M(i)$ together with the recovered decay parameters. In this way at least two decay times which depend on the wavelength of observed light have been found [8]. Residuals $R(i)=M(i)-D(i)$ are plotted across the picture. The PL decay at $2.06 \mathrm{eV}$ of $p$-Si samples show a biexponential decay with lifetimes of $43 \mu \mathrm{s}$ and $164 \mu$ s (sample A) and $32 \mu$ s and $83 \mu$ s (sample B). The multiexponential PL decay times may be associated with phonon assisted transitions from nanocrystallites with different particle sizes.
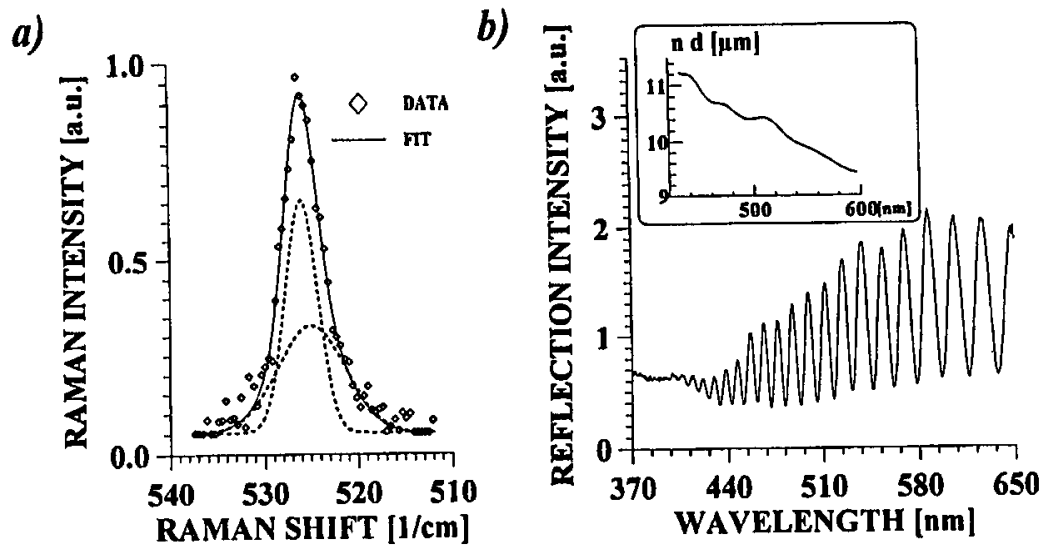

Fig. 2. Typical Raman spectrum of light-emitting porous silicon (sample A); measured (dots) and calculated Raman spectra, dashed line - two gaussians, solid line - calculated line shape (a); reflectivity spectrum of porous silicon (sample B) (b). The inset shows the relative refractive index of porous silicon as a function of the wavelength.

We have also studied dependence of the frequency shift and halfwidth of LO and TO modes in Raman spectra vs. anodization conditions. The typical Raman spectrum (experimental data are shown as dots) for the freshly prepared sample A (porosity $68 \%$ ) is presented in Fig. 2a. Using computer simulation, the Raman spectra were fitted using two gaussians $\left(526 \mathrm{~cm}^{-1}\right.$, FWHW $4 \mathrm{~cm}^{-1}$ and $525 \mathrm{~cm}^{-1}$, FWHW $7.2 \mathrm{~cm}^{-1}$, shown as solid lines). As it can be seen from Fig. 2a the calculated line shape agrees well with the experimental spectrum of porous silicon. The Raman shift to lower energy and line broadening have been observed in 
porous silicon samples and were attributed to the confinement of optical phonons in nanocrystallites [9].

In Fig. 2b we show a reflection spectrum taken on the sample A. To interpret the PLE spectra (Fig. 1a) we assumed that the number of emitted photons is proportional to the number of absorbed excitation photons in small size crystallites of Si. Absorption bands at about $3.4 \mathrm{eV}$ and $4.1 \mathrm{eV}$ in our PLE spectra shift to lower energies with an increase in size crystallites (also see Fig. 2b). In porous silicon samples the crystallites with wide band gap separate the bulk Si regions and the quantum wells are formed with depth and width determined by a crystallites size. The optical energy gap depends on the size of the crystallites $D$ and varies from $1.6 \mathrm{eV}$ to $5 \mathrm{eV}[2,10]$. Because of the small distance between quantum wells the energy levels are broadened due to the coupling and the energy bands are formed. When the size of crystallites decreases the energy gap increases and PLE as well as PL spectra move to the higher energy (Fig. 1a).

The luminescence bands and their relative intensities in the visible region depend also on the preparing conditions. We observed the shift of the PL emission band towards larger photon energies due to the increase in the silicon layer porosity [7]. As the luminescence peak increases in photon energy, the Raman LO mode shifts to lower energy, remaining sharp, and developing into TO and LO modes.

If it is assumed that in porous silicon layers the multi-barrier structure is formed by Si skeleton surrounded by Si nanocrystallites, our Raman scattering, luminescence and reflectivity results can be interpreted in terms of nanocrystallites and related quantization effects.

\section{References}

[1] L.T. Canham, Appl.Phys. Lett. 57, 1046 (1990).

[2] J.P. Proot, C. Delerue, G. Allen, Appl. Phys. Lett. 61, 1948 (1992).

[3] I. Gregora, B. Champagnon, A. Halimaoui, J. Lumin. 57, 73 (1993).

[4] M.G. Berger, U. Klemradt, H. Luth, R. Herino, M. Ligeon, H. Munder, C. Andrzejczak, Thin Solid Films 221, 27 (1992).

[5] R. Tsu, H. Shen, M. Dutta, Appl. Phys. Lett. 60, 112 (1992).

[6] M. Yang, D. Huang, P. Hao, F. Zhang, X. Hou, X. Wang, J. Appl. Phys. 75, 651 (1994).

[7] E. Nossarzewska-Orłowska, A. Brzozowski, Acta Phys. Pol. A 84, 713 (1993).

[8] P.R. Bevington, Data Reduction and Error Analysis for the Physical Sciences, McGraw Hill, New York 1969.

[9] W. Bała, F. Firszt, E. Nossarzewska-Orłowska, A. Brzozowski, B.A. Orłowski, B.J. Kowalski, M. Guziewicz, Acta Phys. Pol. A 84, 761 (1993).

[10] Y.H. Xie, W.L. Wilson, F.M. Ross, J.A. Mucha, F.A. Fitzgerald, J.M. Macaulay, T.D. Harris, J. Appl. Phys. 71, 2403 (1992). 